\title{
Modelamento de Sistemas WLAN em 2,4 GHz para Ambiente Indoor usando Equações Parabólicas
}

\author{
Fátima Nazaré Baraúna Magno, João Furtado de Souza, Klaus Cozzolino, Jessé Carvalho Costa, Gervásio Protásio \\ dos Santos Cavalcante \\ Universidade Federal do Pará - Belém - Pará - Brasil
}

\begin{abstract}
Resumo - Este trabalho apresenta o cálculo da perda de propagação em ambiente indoor, utilizando o método de equações parabólicas, cuja solução é obtida com a aplicação do esquema implícito de diferenças finitas de Crank-Nicolson. A validação do modelo foi feita realizando uma campanha de medições em prédio da Engenharia Elétrica e de Computação da Universidade Federal do Pará, para uma freqüência de 2,442 GHz, utilizada em sistemas WLAN. O modelo proposto é, ainda, comparado a alguns modelos da literatura. É considerada a propagação em $15^{0}$ na direção paraxial; assim como o índice de refração complexo. $O$ desvio padrão encontrado foi, em média, de 2,27 dB.
\end{abstract}

Palavras-Chave - Equação parabólica, Esquema de diferenças finitas, Indoor, Perda de propagação.

\begin{abstract}
This paper presents the calculation of the path loss in indoor environment, using the parabolic equation; the implicit finite difference scheme of the Crank-Nicholson type was applied in order to solve the parabolic equation. To validate the model a campaign of measurements was carried out in an Electric Engineering and Computation building in the Federal University of Pará. A $2.442 \mathrm{GHz}$ frequency was used. The proposed model is compared with the some models of the literature. The propagation is considered in $15^{\circ}$ the paraxial direction; as well as a complex refractive index was considered. The standard deviation for measured versus predicted signal is within $2.27 \mathrm{~dB}$.
\end{abstract}

Keywords — Finite difference scheme, Indoor, Parabolic equation, Path loss

Fátima N. B. Magno, João F. de Souza, Klaus Cozzolino, Departamento de Física; Jessé C. Costa, Departamento de Geofísica; Gervásio P. S. Cavalcante, Departamento de Engenharia Elétrica. Universidade Federal do Pará, Belém, Pará, Brasil. E-mails: fnbm@ufpa.br, furtado@ufpa.br, cozolino@ufpa.br, jesse@ufpa.br, gervasio@ufpa.br.

\section{I.INTRODUÇÃO}

Nos últimos anos, o avanço do sistema de comunicação digital sem fio foi notável. A comunicação sem fio em recinto fechado (indoor) é, hoje, um dos campos com mais rápido desenvolvimento. Há uma procura crescente em modelos de propagação que proporcionem um modelamento do sinal eficiente e preciso.

A simulação da propagação de ondas eletromagnéticas em ambientes indoor é uma tarefa importante para redes sem fio. Embora seja estudado há mais de 15 anos, o problema de encontrar o equilíbrio certo entre a carga computacional e a precisão permanece um assunto aberto. Novas aplicações e tecnologias tais como as redes ad hoc ou redes de sensores, transmissão através de múltiplas antenas, etc., buscam novos modelos de propagação específicos [1].

O estudo da radiopropagação nestes ambientes é muito importante para a determinação das perdas que ocorrem no interior de edifícios, pois qualquer objeto, na faixa de frequiência considerada, pode atuar como uma fonte de interferência para as ondas eletromagnéticas que se propagam nas células indoor. Desta maneira, o modelamento matemático de tal ambiente é extremamente complexo e o conhecimento da distribuição do campo é crucial [2].

Dois tipos de modelos de propagação são muito usados para picocélulas indoor, os empíricos e os determinísticos. Os primeiros estão baseados em fórmulas muito simples e diretas, fáceis de aplicar, mas por outro lado estes modelos proporcionam precisão local-específica pobre e não podem predizer parâmetros da banda larga do canal de comunicação. Os modelos determinísticos seguem princípios físicos de propagação de ondas eletromagnéticas os mais populares são o ray tracing e a solução numérica das equações de Maxwell.

O método de equações parabólicas (EP) fornece uma excelente combinação de precisão e eficiência para muitos problemas de propagação de ondas eletromagnéticas. Neste método a aproximação é feita desprezando-se o retro espalhamento. Este método tem apresentado vantagem para o fenômeno da propagação indoor, principalmente quanto à 
redução do tempo computacional gasto, quando o principal interesse é a determinação das características do desvanecimento do canal, além de requerer muito menos recurso computacional que o método totalmente elíptico [3].

O modelo apresentado neste trabalho é baseado no método de equações parabólicas para o cálculo da perda de propagação utilizando o esquema de diferenças finitas de Crank-Nicolson, considerando a propagação de ondas eletromagnéticas em ambiente indoor. Objetiva-se calcular o campo recebido em um prédio de dois andares, o Anexo II do Laboratório de Engenharia Elétrica e de Computação (LEEC) da Universidade Federal do Pará (UFPA). Considera-se aqui o índice de refração complexo e com valores diferenciados, dependendo da permissividade relativa e da condutividade, para os vários materiais usados na construção do ambiente estudado. A freqüência utilizada foi de 2,442 GHz, que é a usada em WLAN, e foi considerada a propagação em $15^{0}$ na direção paraxial. Para a validação do modelo proposto foi realizada uma campanha de medições onde o sistema transmissor foi fixado no corredor do pavimento térreo e o sistema receptor foi posicionado nos vários pontos de medição distribuídos pelos andares térreo e superior do ambiente de teste. A curva obtida pelo método proposto foi, então, comparada aos dados obtidos experimentalmente e com três modelos existentes na literatura.

Este trabalho está organizado como segue: o modelo teórico, método de equações parabólicas (EP) e sua resolução pelo esquema de diferenças finitas de CrankNicolson, é descrito na secção II; na secção III é feita a descrição do ambiente utilizado; a perda de propagação calculada via equações parabólicas e dados experimentais é mostrada na seção IV; na seção V apresenta-se os modelos da literatura com os quais são feitas as comparações; os resultados dos cálculos via equações parabólicas, modelos e dados experimentais, com suas respectivas figuras e tabelas representativas, são apresentados na secção VI e na seção VII, a conclusão.

\section{O MÉTODO TEÓRICO}

Nesta seção desenvolve-se inicialmente o método de equações parabólicas que foi introduzido por Leontovich e Fock, em 1940, para estudar problemas de difração e radiopropagação.

A equação de onda escalar em três dimensões, denominada equação de Helmholtz, para uma componente $\psi$ do campo eletromagnético é dada por

$$
\left(\nabla^{2}+k^{2} n^{2}\right) \psi(x, y, z)=0
$$

sendo $k=2 \pi$ / $\lambda$ o número de onda no vácuo e $n$ o índice de refração do meio [4].

Se as equações de Maxwell são expressas em coordenadas cilíndricas e todas as fontes estão no eixo $z$, então todas as componentes dos campos são independentes do ângulo azimutal. Objetivando-se simplificar as equações de Maxwell, pode-se assumir esta simetria, o que reduz o problema para coordenadas cartesianas ortogonais, possibilitando que a equação de Helmholtz seja decomposta em dois casos - polarização vertical e horizontal [5]. A equação de onda reduz-se, então, a

$$
\frac{\partial^{2} \psi}{\partial z^{2}}+\frac{\partial^{2} \psi}{\partial x^{2}}+\frac{1}{x} \frac{\partial \psi}{\partial x}+k^{2} n^{2} \psi=0
$$

que é uma equação diferencial parcial elíptica contendo segundas derivadas em relação a $x$ e $z$.

Escrevendo-se $\psi$ da forma [6]

$$
\psi(x, z)=u(x, z) \frac{e^{i k x}}{\sqrt{x}}
$$

onde u representa o campo elétrico. Substituindo (3) em (2) obtém-se

$$
\frac{\partial^{2} u}{\partial z^{2}}+\frac{\partial^{2} u}{\partial x^{2}}+2 i k \frac{\partial u}{\partial x}+k^{2}\left(n^{2}-1+\frac{1}{(2 k x)^{2}}\right) u=0
$$

Como é interessante estudar-se locais distantes da fonte, então $k x \gg 1$, o que permite desprezar-se o termo $1 /(2 k x)^{2}$. Tomando-se pequenas variações para $u$, o que implica em

$$
\left|\frac{\partial^{2} u}{\partial x^{2}}\right| \ll 2 k\left|\frac{\partial u}{\partial x}\right|
$$

reduz-se (4) à equação parabólica padrão (EPP), através da qual calcula-se o campo elétrico [6]

$$
\frac{\partial^{2} u}{\partial z^{2}}+2 i k \frac{\partial u}{\partial x}+k^{2}\left(n^{2}(x, z)-1\right) u=0
$$

Para encontrar-se (6) foi feita a aproximação válida para pequenos ângulos, no intervalo $15-25^{0}$ com a horizontal, e tomou-se a propagação progressiva da onda [7].

Discretizando-se (6) em $x$ e $z$ através do esquema de diferenças finitas de Crank-Nicolson [8], fazendo-se $\xi_{m}=\left(x_{m-1}+x_{m}\right) / 2$ o ponto médio na solução de $x_{\mathrm{m}-1}$ para $x_{\mathrm{m}}, \quad u_{j}^{m}=u\left(x_{m}, z_{j}\right), \quad b=4 i k\left(\Delta z^{2} / \Delta x\right) \quad$ e $a_{j}^{m}=k^{2}\left(n^{2}\left(\xi_{m}, z_{j}\right)-1\right) \Delta z^{2}$, obtém-se [4]

$$
u_{j}^{m}\left(-2+b+a_{j}^{m}\right)+u_{j+1}^{m}+u_{j-1}^{m}=u_{j}^{m-1}\left(2+b-a_{j}^{m}\right)-u_{j+1}^{m-1}-u_{j-1}^{m-1}
$$

\section{O AMBIENTE DE TESTE}

O objetivo do modelo proposto, desenvolvido para estudar a propagação de ondas eletromagnéticas em ambientes indoor, é calcular o campo elétrico dentro do Anexo II do Laboratório de Engenharia Elétrica e de Computação da Universidade Federal do Pará.

O ambiente de teste é composto de um prédio de dois andares, construído recentemente, e por isto não estando, até o momento, nem mobiliado nem habitado, de modo que se devem considerar apenas os materiais usados em sua construção, tais como paredes em alvenaria e divilux, portas em madeira, ferro e divilux, e janelas em vidro com esquadrias de alumínio. O prédio em questão foi projetado para que em seu interior funcionem laboratórios, salas de professores, etc. As diferentes estruturas geométricas e os vários materiais envolvidos interferem na propagação da 
onda eletromagnética, ocasionando redução no sinal recebido, quando comparado ao transmitido.

As plantas baixas apresentadas abaixo (ver Figs. 1 e 2) correspondem aos dois pavimentos do prédio onde foi realizada a campanha de medições. Ressalta-se que a distribuição de móveis é hipotética, já que o prédio ainda não foi mobiliado.

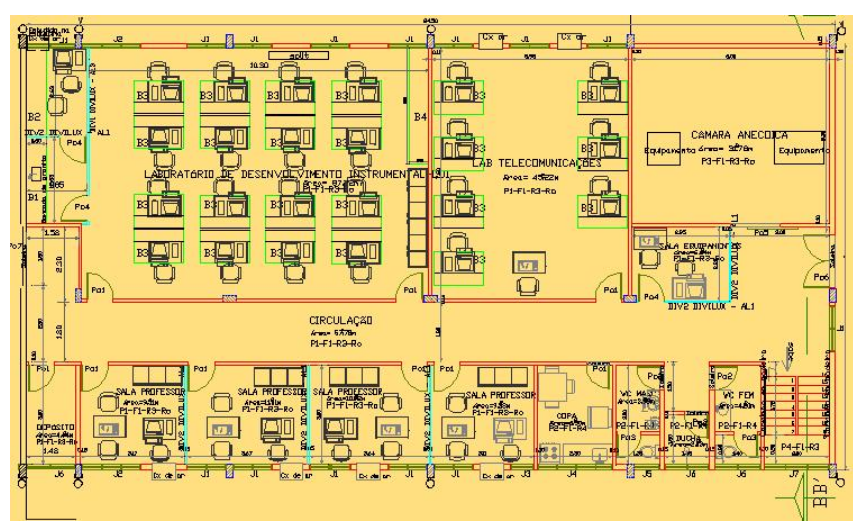

Fig. 1. Planta baixa do pavimento térreo do ambiente de teste

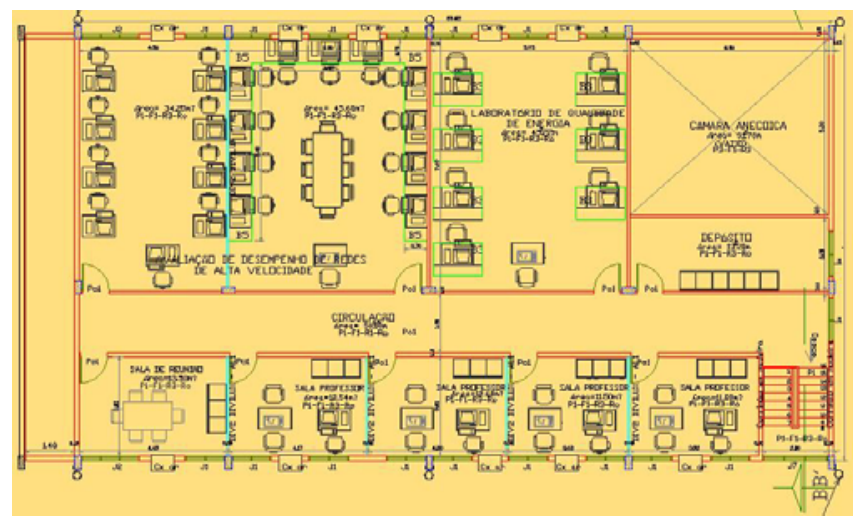

Fig. 2 . Planta baixa do pavimento superior do ambiente de teste

No ambiente de teste, observa-se a semelhança entre os corredores existentes no pavimento térreo e superior, modificando-se apenas a disposição das portas e janelas e salas existentes ao longo deles. Na Fig.3 mostra-se o corredor existente no pavimento térreo.

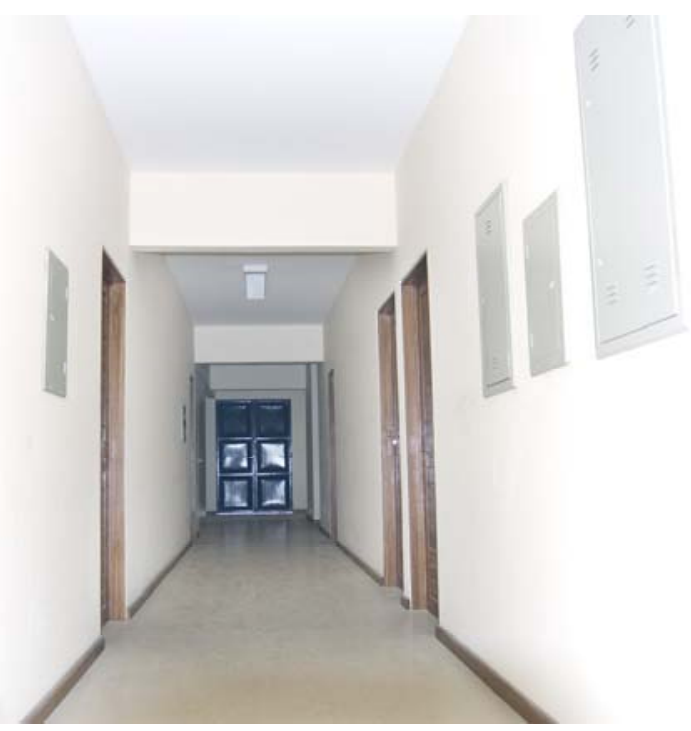

Fig.3 . Corredor do pavimento térreo
IV. PERda de PropagaÇÃo CALCUladA VIA EQUAÇÕES PARABÓLICAS E EXPERIMENTALMENTE

Para a simulação via equações parabólicas (EP) considerou-se o sinal transmitido por uma onda plana polarizada verticalmente incidindo sobre o corredor em linha direta, com freqüência de 2,442 GHz; foi escolhida a direção paraxial, com aproximação para pequenos ângulos, até $15^{\circ}$. Consideraram-se as dimensões de comprimento e largura dos pavimentos térreo e superior. Na implementação foi utilizado um programa FORTRAN, para resolução de (7), e para os gráficos utilizou-se o Matlab 7.

Na campanha de medições foram, inicialmente, marcados no chão os pontos onde a medição seria realizada, totalizando 21 pontos no andar superior e 25 no pavimento térreo. Escolheram-se, então, duas paredes próximas para que os pontos-medidas fossem posicionados na planta baixa do prédio, utilizando o programa AUTOCAD. Foi, após isto, posicionado um ponto de acesso na entrada do corredor do andar térreo. Um notebook foi usado como receptor de chamadas VoIP, conectado a um analisador de protocolo; as chamadas foram realizadas através do CallGen323, desenvolvido pelo projeto OpenH323 [9]. Um outro notebooks sobre um carrinho (que percorreu os dois pavimentos do prédio em estudo) fazia a medida da potência recebida em cada ponto através do Network (c) Stumbler software [10].

Foram armazenados os seguintes parâmetros: potência recebida (que será usada neste trabalho para determinar a perda de propagação), distância entre o transmissor e o receptor, e alguns parâmetros referentes à qualidade do serviço (jitter, atraso de pacotes e PMOS) que serão usados em trabalhos futuros.

Os materiais empregados na construção do prédio apresentam características diferentes, sendo necessário que se utilizem os valores das constantes eletromagnéticas de cada material. A Tabela I apresenta os valores da permissividade relativa e da condutividade dos vários materiais empregados na construção do prédio.

TABELA I

PERMISSIVIDADE RELATIVA E CONDUTIVIDADE DOS MATERIAIS

\begin{tabular}{|c||c|c|}
\hline Materiais & Permissividade Relativa & Condutividade (S/m) \\
\hline \hline Alvenaria [11] & 5,18 & $0,032-0,040$ \\
\hline Madeira [11] & 3,00 & 0,01 \\
\hline PVC [12] & 2,26 & 0,00 \\
\hline Ferro [12,13] & 12,00 & $1,03 \times 10^{-7}$ \\
\hline
\end{tabular}

O cálculo do índice de refração foi feito utilizando-se [14]

$$
n=\left[\varepsilon_{r}+\frac{i \rho}{2 \pi f \varepsilon_{0}}\right]^{1 / 2}
$$

onde $\varepsilon_{\mathrm{r}}$ é a permissividade relativa, $\rho$ a condutividade $(\mathrm{S} / \mathrm{m}), \quad f$ a freqüência $(\mathrm{Hz})$ e $\varepsilon_{0}$ é a permissividade no vácuo (F/m).

A perda de propagação foi calculada por

$$
L(d B)=36,57+20 \log _{10} f+20 \log _{10}\left|u_{0}\right|-20 \log _{10}|u|-G_{T}-G_{R}
$$


sendo $u_{0} \quad$ o campo na distância de referência $\left(d_{0}\right), \quad u \quad$ o campo recebido, $f$ a freqüência expressa em $\mathrm{GHz}$ e $G_{T}$ e $G_{R}$ os ganhos das antenas transmissora e receptora em $\mathrm{dB}$, respectivamente.

\section{MOdelos PARA A PERdA DE PROPAGAÇÃO}

A perda de propagação, encontrada através do método proposto, foi comparada com alguns modelos existentes na literatura descritos a seguir.

\section{A. Modelo Dependente da Distância}

Os modelos de propagação, teóricos e experimentais, indicam que a potência do sinal recebida decresce logaritmicamente com a distância, tanto para ambiente outdoor como para indoor. A perda de propagação é expressa como uma função da distância como [15]

$$
P L(d B)=P L\left(d_{0}\right)+10 n \log \left(d / d_{0}\right)
$$

sendo $P L\left(d_{0}\right)=20 \log \left(4 \pi d_{0} / \lambda\right)$, em $\mathrm{dB}$, a perda de propagação referente à distância de referência $d_{0}(1 \mathrm{~m}), n$ o expoente de perda de propagação que indica a razão com que a perda de propagação aumenta com a distância, e $d$ a distância de separação entre o transmissor e o receptor.

\section{B. Modelo do Fator de Atenuação de Piso (FAP)}

Como uma alternativa à (13), quando há um ou mais pisos separando o transmissor e o receptor, um fator de atenuação, FAP (em dB), que é uma função do número de pisos e do tipo de edifício, pode ser adicionado à perda de propagação [16]

$$
P L(d B)=P L\left(d_{0}\right)+10 n \log \left(d / d_{0}\right)+F A P(d B)
$$

As perdas entre os pisos de um edifício são determinadas pelas dimensões externas e materiais do edifício, assim como pelo material utilizado para a construção do piso e áreas que o cercam.

\section{Modelo de Simon Wong, Francis Lau e Chi Tse}

No ambiente estudado pelos autores, foi percebida a vantagem em se adotar uma aproximação empírica por considerar todos os fatores que influenciam a propagação da onda. O modelo de propagação é baseado na perda de propagação descrito em [15]. Além disso, os efeitos de desvanecimento em pequena escala podem também ser caracterizados através de um parâmetro de desvanecimento devido ao sombreamento, $\chi_{\sigma}$, dado por [17]

$$
P L(d B)=P L\left(d_{0}\right)+10 n \log \left(d / d_{0}\right)+\chi_{\sigma}
$$

sendo $\chi_{\sigma}$ uma variável aleatória (em dB) de uma distribuição Gaussiana de média zero e desvio padrão $\sigma$ (em dB). Os valores de $n$ e $\sigma$ dependem da propagação no ambiente onde foram tomadas as medidas.

\section{Modelo de N. Yarkoni e N. Blaunstein}

Neste modelo é apresentada uma aproximação semiempírica e um modelo analítico para a predição da perda de propagação total em ambientes indoor (entre pisos e ao longo de corredores). Os autores consideram as propriedades físicas do ambiente e os parâmetros da atenuação total obtidos através das medidas experimentais. É sugerido, então, para a perda de propagação a seguinte fórmula [18]

$$
P L_{\text {total }}=P L\left(d_{0}\right)+10 n \log \left(d / d_{0}\right)+P L_{\text {desvanecimento }}
$$

sendo

$$
P L_{\text {desvanecimento }}=P L_{S F}+P L_{F F}
$$

Aqui $P L_{S F}=10 \log \sigma_{S F}$, onde $\sigma_{S F}$ é uma distribuição lognormal de uma variável aleatória de média zero e desvio padrão $\sigma_{L}$ (em $\left.\mathrm{dB}\right) ; \quad P L_{S F}$ é uma atenuação provocada pela difração das ondas que se propagam (também chamada de efeito do sombreamento devido às obstruções). Já $P L_{F F}=10 \log \sigma_{F F}$, sendo $\sigma_{F F}$ uma distribuição de Rice de uma variável aleatória com desvio padrão $\sigma$ (em $\mathrm{dB}$ ), e é decorrente do fenômeno das múltiplas reflexões e espalhamentos causado pela localização das obstruções que circundam os terminais das antenas.

\section{RESULTADOS NUMÉRICOS}

Inicialmente, foi obtido o mapa da perda de propagação, em $\mathrm{dB}$, versus comprimento e largura do ambiente, em metros, para os pavimentos térreo e superior, calculada via equações parabólicas, mostrando os locais onde a perda é maior ou menor (ver Figs. 4 e 5).

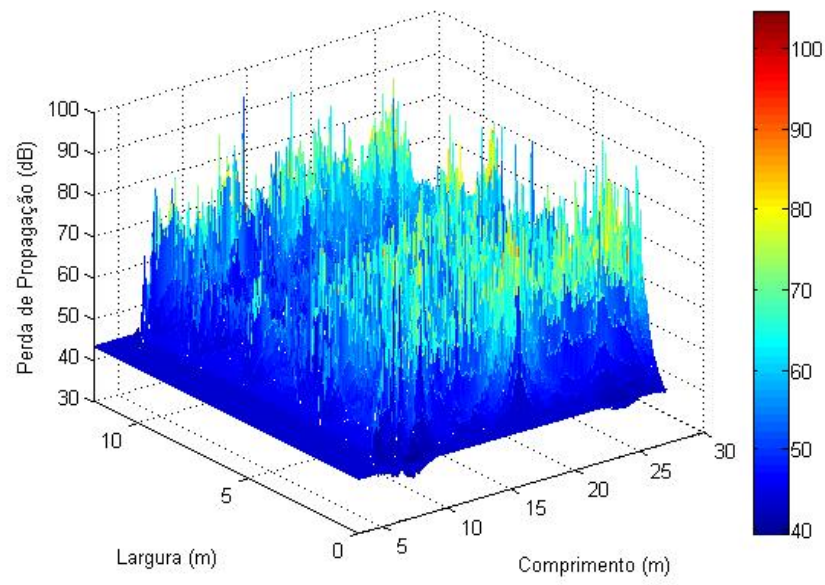

Fig. 4. Perda de propagação para o pavimento térreo

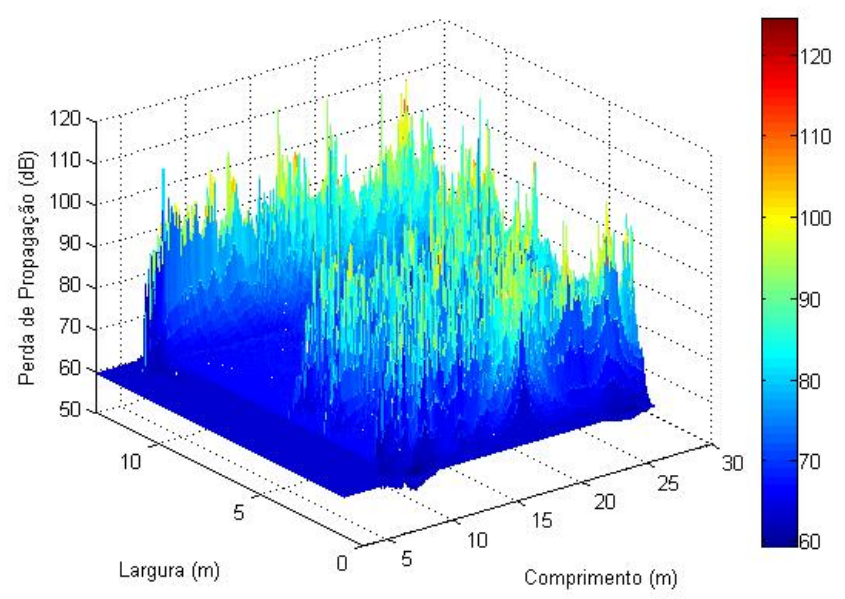

Fig. 5. Perda de propagação para o pavimento superior 
A Fig. 6 mostra as curvas da perda de propagação, em dB, versus distância, em metros, para o cálculo usando o método de equações parabólicas (EP), modelo dependente com a distância, modelo de Simon Wong, Francis Lau e Chi Tse, modelo de N. Yarkoni e N. Blaunstein e valores medidos experimentalmente, no pavimento térreo. Neste cenário foi considerada a propagação em visada direta, observando-se boa concordância entre o método proposto, os dados experimentais e os outros modelos.

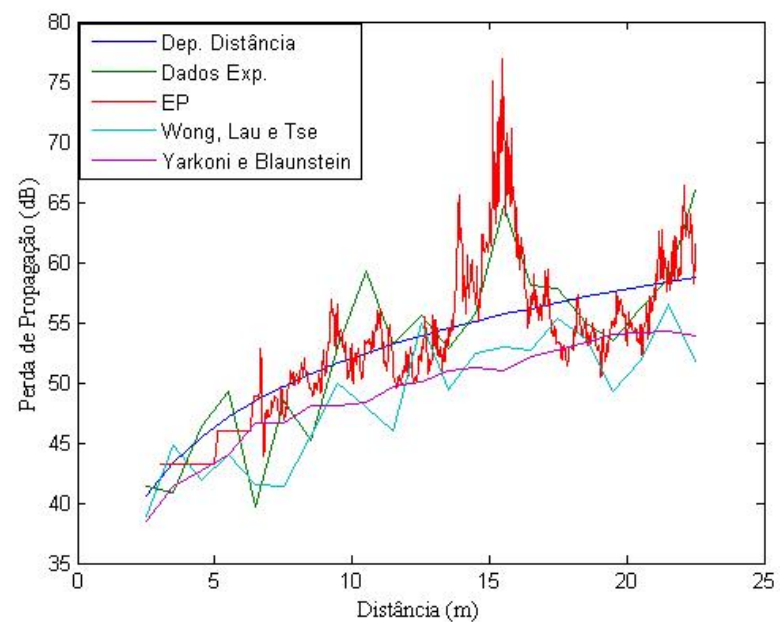

Fig. 6. Perda de propagação versus distância no pavimento térreo calculada via EP, experimentalmente e através dos modelos dependente da distância, de Wong, Lau e Tse, e de Yarkoni e Blaunstein

A Fig. 7 mostra as curvas da perda de propagação, em dB, versus distância, em metros, para o cálculo usando o método de equações parabólicas (EP), modelo Fator de Atenuação de Piso (FAP), modelo de Simon Wong, Francis Lau e Chi Tse, modelo de N. Yarkoni e N. Blaunstein e dados experimentais, no pavimento superior. Novamente, pode-se observar a boa concordância entre o método de equações parabólicas e os outros modelos, assim como com as medidas experimentais.

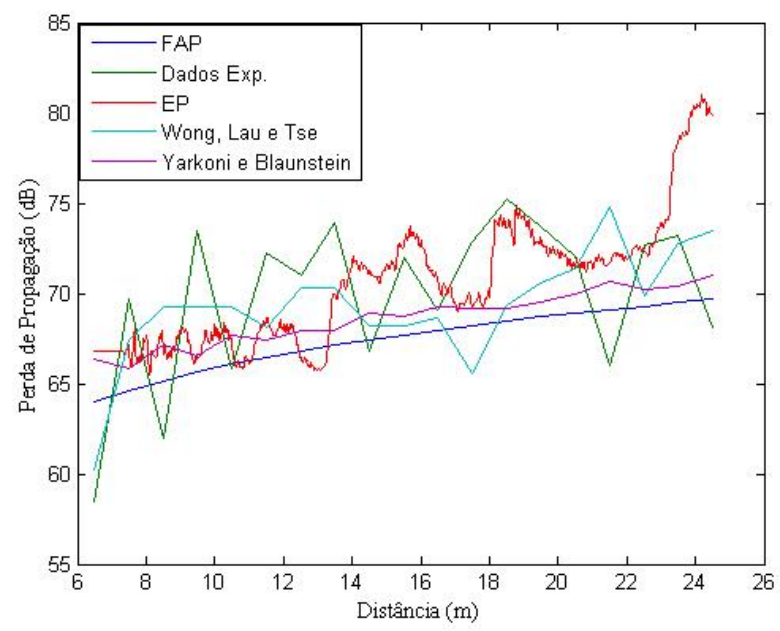

Fig. 7. Perda de propagação versus distância no pavimento superior calculada via EP, experimentalmente e através dos modelos FAP, de Wong, Lau e Tse, e de Yarkoni e Blaunstein

Analisando-se as Figs. 6 e 7, nota-se uma diferença de, aproximadamente, $19 \mathrm{~dB}$ para uma mesma posição, ao comparar-se os dois pavimentos, o que se deve ao piso de concreto, com aproximadamente $20 \mathrm{~cm}$ de espessura, existente entre a antena transmissora e a antena receptora.

A Tabela II mostra o expoente de perda de propagação para os pavimentos térreo e superior. Percebe-se que este fator depende do ambiente e do cenário [19].

TABELA II

EXPOENTE DE PERDA DE PROPAGAÇÃO

\begin{tabular}{|c||c|c|}
\hline \multirow{2}{*}{\multicolumn{1}{|c||}{ Pavimento }} & \multicolumn{2}{c|}{ Expoente de Perda de Propagação } \\
\cline { 2 - 3 } & Via EP & $\begin{array}{c}\text { Dados } \\
\text { Experimentais }\end{array}$ \\
\hline \hline Térreo & 1,9 & 2,2 \\
\hline Superior & 0,98 & 1,1 \\
\hline & 1,44 & 1,65 \\
\hline
\end{tabular}

A Tabela III fornece o erro médio, desvio padrão e erro rms para os pavimentos estudados no ambiente de teste, comparados às medidas experimentais e aos modelos da literatura.

TABELA III

CÁLCULO DO ERRO MÉDIO, DESVIO PADRÃO E ERRO RMS

\begin{tabular}{|c|c|c|c|c|}
\hline Pavimento & Modelo & $\begin{array}{l}\text { Erro } \\
(\mathrm{dB})\end{array}$ & $\begin{array}{l}\text { Desvio Padrão } \\
\text { (dB) }\end{array}$ & $\begin{array}{l}\text { Erro rms } \\
\text { (dB) }\end{array}$ \\
\hline \multirow{4}{*}{ Térreo } & EP & 2,74 & 2,19 & 3,51 \\
\hline & $\begin{array}{l}\text { Dep. Distância } \\
\text { [15] }\end{array}$ & 3,26 & 2,98 & 4,41 \\
\hline & $\begin{array}{c}\text { Wong, Lau e Tse } \\
\text { [17] }\end{array}$ & 4,78 & 2,83 & 5,56 \\
\hline & $\begin{array}{c}\text { Yarkoni e } \\
\text { Blaunstein [18] }\end{array}$ & 3,94 & 3,53 & 5,29 \\
\hline \multirow{4}{*}{ Superior } & EP & 2,96 & 2,33 & 3,77 \\
\hline & FAP [16] & 3,44 & 2,03 & 4,00 \\
\hline & $\begin{array}{l}\text { Wong, Lau e Tse } \\
\text { [17] }\end{array}$ & 3,09 & 2,89 & 4,23 \\
\hline & $\begin{array}{c}\text { Yarkoni e } \\
\text { Blaunstein [18] }\end{array}$ & 3,35 & 1,85 & 3,83 \\
\hline
\end{tabular}

\section{VII.CONCLUSÃO}

Neste trabalho foi utilizado um modelo determinístico, o método de equações parabólicas, para o estudo da perda de propagação em um ambiente indoor.

Notou-se que a utilização deste modelo tem grande rapidez no processamento dos dados. Isto é possível pelo fato de que, na resolução do sistema, utiliza-se o esquema implícito de diferenças finitas do tipo Crank-Nicolson onde, em lugar de ter-se que resolver uma matriz com todos os elementos diferentes de zero, resolve-se uma tridiagonal; este esquema foi escolhido por ser incondicionalmente estável.

Para o cálculo do índice de refração foram consideradas as partes real e imaginária, o que aumenta a precisão do modelo proposto. 
Os valores encontrados foram, em média, de 2,85 dB para o erro, 2,27 dB para o desvio padrão e, de 3,65 dB para o erro rms. Estes resultados mostram o bom comportamento do método de equações parabólicas no estudo da perda de propagação em ambientes indoor, comparado à outros modelos da literatura e medidas experimentais.

Como trabalho futuro, pretende-se fazer o estudo da perda de propagação com o ambiente mobiliado e habitado.

Atualmente, estão sendo feitas simulações aumentando o ângulo de propagação para até $90^{\circ}$ com a direção paraxial e avaliando a perda de propagação com a existência de redes interferentes.

\section{REFERENCES}

[1] J. Gorce, K. Jaffrès-Runser and G. de la Roche, "Deterministic Approach for Fast Simulations of Indoor Radio Wave Propagation”, IEEE Transactions on Antennas and Propagation, Vol. 55, No. 3, March 2007.

[2] S.Grubisic, W. P. Carpes, C. B. Lima and P. Kuo-Peng, "Ray-Tracing Propagation Model Using Image Theory With a New Accurate Approximation for Transmitted Rays Through Walls”, IEEE Transactions on Magnetic, Vol. 42, No. 4, April 2006.

[3] E. T. Küsel, W. L. Siegmann and M. D. Collins, “A single-scattering for large contrasts in elastic layers”, Journal Acoustical Society of America, 121 (2), February 2007.

[4] M. Levy, "Parabolic Equation Methods for Electromagnetic Wave Propagation”, the Institution of Electrical Engineers, London, pp. 440, 2000.

[5] J. Kuttler, "Differences Between the Narrow-Angle and Wide-Angle Propagators in the Split-Step Fourier Solution of the Parabolic Wave Equation”, IEEE Transactions on Antennas and Propagation, Vol. 47, No. 7, July 1999.

[6] K. H. Craig and M.F. Levy, "Parabolic Equation Modeling of the Effects of Multipath and Ducting on Radar Systems”, IEE Proceedings-F, Vol. 138, No. 2, April 1991.

[7] A. Z. Hyaric, "Wide-Angle Nonlocal Boundary Conditions for the Parabolic Wave Equation”, IEEE Transactions on Antennas and Propagation, Vol. 49, No. 6, June 2001.

[8] G. D. Smith, "Numerical Solution of Partial Differential Equations Finite Difference Methods”, Oxford: Clarendon, 3rd edition, pp. 1138, 1984.

[9] http://www.openh323.org/ accessed in 12/05/2006.

[10] http://www.netstumbler.com/ accessed in 12/19/2006.

[11] D. I. Axiotis and M. E. Theologou, "2 GHz Outdoor to Indoor Propagation at high Elevation Angles”, IEEE, PIMRC 2002.

[12] C. A. Balanis, “Advanced Engineering Electromagnetic”, John Wiley \& Sons, United States of America, pp. 50-62, 1989.

[13] W. H. Hayt, "Engineering Electromagnetic”, McGraw-Hill, New York, $4^{\text {th }}$ edition, pp. 508-509, 1981.

[14] R. K. Wangsness, "Electromagnetic Fields", John Wiley \& Sons, United States of America, pp. 34 - appendices, 1979.

[15] T. S. Rappaport, "Wireless Communications: Principles and Practice", Prentice Hall PTR, Upper Saddle River, NJ, United States of America, 2nd edition, 2002.

[16] S. Seidel, and T. Rappaport, "914 MHz Path Loss Prediction Models for Indoor Wireless Communications in Multifloored Buildings”, IEEE Transactions on Antennas and Propagation, Vol. 40, No. 2, February 1992.

[17] S. S. M. Wong, F. C. M. Lau and C. K. Tse, "Propagation Characteristics of UWB Radio in a High-Rise Apartment”, ICACT, pp. 914-918, 2006.

[18] N. Yarkoni and N. Blaunstein, "Prediction of Propagation Characteristics in Indoor Radio Communication Environments", Progress in Electromagnetics Research, PIER 59, pp. 151-174, 2006.

[19] C. C. Chong, Y. Kim and S. Lee, "Statistical Characterization of the UWB Propagation Channel in Various Types of High-Rise Apartments”, IEEE Communications Society, pp. 944-949, 2005. 